\title{
Screening Recommendations for Asymptomatic Carotid Artery Disease and Role of Carotid Intima-Media Thickness Evaluation: A Literature Review Dedy Pratama ${ }^{1}$, Fajar Satria Rahmaditya*1
}

Background: Asymptomatic carotid artery disease should be treated as soon as possible before the atherosclerotic disease progressed into ischaemic stroke. Diagnostic screening by measuring intima-media thickness (IMT) of carotid artery using ultrasound is one possible way to evaluate earlier the progression compared to standard stenosis evaluation. Not only the necessity of carotid screening is still questioned by some experts, current guidelines also show conflicting results in certainty whether we should perform the carotid screening or not. It is necessary to conduct a literature review of relevant recommendations from the latest clinical practice guidelines (CPGs).

Method: The CPGs on the management of asymptomatic carotid artery disease published before October 2020 were retrieved from online databases and the rigor of guideline development assesed by using the Appraisal of Guidelines Research and Evaluation II (AGREE-II) instrument. The quality of CPGs was assessed and categorized, and their reported evidences were also evaluated.

Results: Six eligible CPGs were included. Five of the CPGs recommends carotid screening for highly selective patient who has morbidity regarding atherosclerotic disease manifested in other organs. One CPG against the screening as further treatment considered cause more harms, although the recommendations mainly based on hypothetical outcomes and the CPG itself categorized into moderate quality.

Conclusion: Our critical review on those CPGs showed that screening for asymptomatic carotid artery disease should be performed for specific high-risk populations of atherosclerotic disease. Although IMT evaluation could be a promising technique, current guidelines didn't show any recommendation regarding the usage comparing standard ultrasound evaluation.

Keywords: Asymptomatic carotid artery disease; ultrasound screening; intima-media thickness; clinical practice guidelines

https://doi.org/10.36864/jinasvs.2021.1.010

*Corresponding author: fajar.satria91@ui.ac.id

${ }^{1}$ M.D. Vascular and Endovascular Division, Department of Surgery, Cipto Mangunkusumo Hospital - Faculty of Medicine, Universitas Indonesia, Jakarta, Indonesia

\section{INTRODUCTION}

Carotid artery disease, defined as blockade or narrowing of lumen of the carotid arteries caused by cholesterol deposition and atherosclerosis, is one of important etiology of ischaemic stroke. Carotid artery disease is present when there is carotid artery stenosis $>50 \%$ of lumen size of common carotid artery (CCA) on either side or thickening of IMT $\geq 1$ $\mathrm{mm}$, usually found by ultrasonography examination.1,2 Carotid artery disease should be managed early as global burden of ischaemic stroke increased steadily over time, showed in increasing number of annual mortality, morbidity, as well as direct and indirect economic losses caused by it. Global prevalance of stroke is estimated in $11.3 \%$ of total death, and $73-86 \%$ of them are ischaemic stroke.3,4 Prevention measure, including diagnostic screen in carotid artery disease treatment could be a key role in managing ischaemic stroke.

Patient who already suffered from ischaemic stroke, focal neurologic deficit, as well as transient ischaemic attack due to emboli in cerebral artery, which categorized into symptomatic carotid disease are relatively easy to be diagnosed due to clarity of the symptoms. On the other hand, asymptomatic disease without any neurologic symptoms in the last six months, are more challenging to be identified. ${ }^{5}$ Patient categorization which only in certain criteria we should perform routine screening to identify carotid artery disease to be treated further is needed. Early IMT evaluation could possibly asses carotid artery disease in earlier stage as plaque and stenosis present when IMT exceeded $1.5 \mathrm{~mm}(1.2-1.9 \mathrm{~mm}$ range in some studies). ${ }^{6}$ 
Table 1. Six domains of AGREE-II instrument

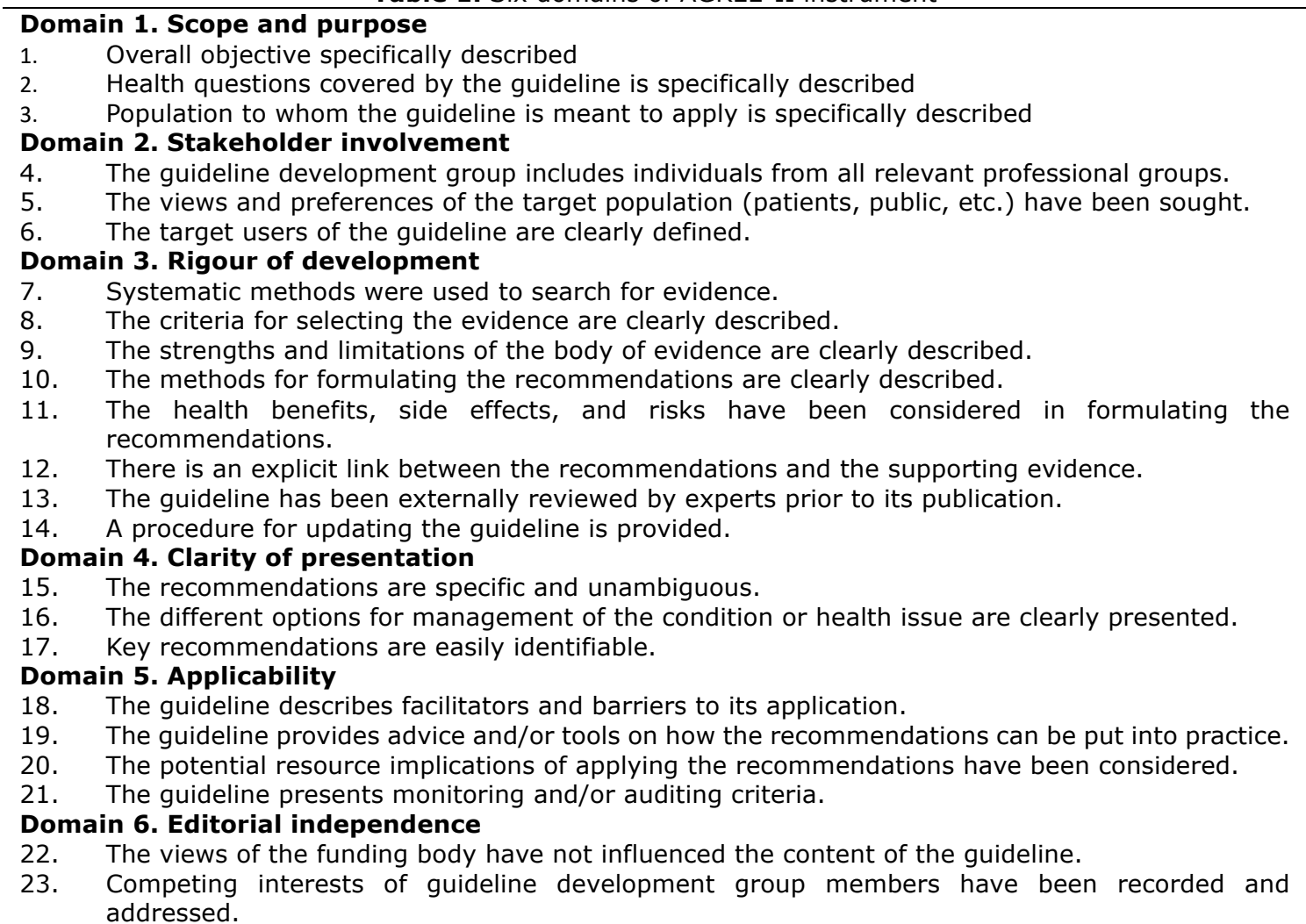

The important steps to do are adapting and appraising current global clinical practice guidelines (CPGs). Several notable professional organizations have developed CPGs containing recommendations for managing asymptomatic carotid disease, including routine screening in certain population. However, current available CPGs contains several contradictory recommendations. For examples, United States Prevention Service Task Force (USPSTF) strongly against the carotid screening as current pathway of screening followed by management for positive disease cause significantly more harm for asymptomatic population. ${ }^{7}$ On the other hand, screening is an important step to identify early carotid artery disease so management of the disease can be started early so ischaemic stroke can be prevented, as recommended by another CPGs.

The quality of these CPGs is of concerned. The appraisal of guidelines, research, and evaluation (AGREE) instrument is a tool for assessing the quality of guidelines. AGREE-II, the updated version, is currently a standard instrument in the evaluation and development of CPGs. ${ }^{8}$ Using the AGREE-II instrument, we reviewed and assessed the quality and consistency of the recommendations of CPGs on the recommendation regarding the importance of carotid screening.

\section{METHOD}

We conduct literature review of CPGs, collected in October 2020 from United States' Agency for Healthcare Research and quality (AHRQ), United Kingdom's National Institute for Health and Care Excellence (NICE), Cochrane Library, National Library of Medicine / MEDLINE, EBSCO and Proquest. The following terms and Boolean operators were used in $\mathrm{MeSH}$ and free-text searches: asymptomatic, carotid artery disease OR carotid stenosis, ultrasound OR intima-media thickness OR risk factor scoring, screening or guidelines.

All the CPGs discuss the management of asymptomatic carotid artery disease, especially screening of possible cases in high-risk population. We didn't apply language or timeline restrictions for online searching. When more than one set of guidelines was produced by the same professional body, only the most recently issued was considered. Critical appraisal for these guidelines then performed aided by AGREE-II instrument, developed by AGREE Next Steps Consortium in 2013, by evaluating semiquantitatively these 23 points including in 6 domains (table 1 ). ${ }^{8}$

Classification then made to stratify the quality of those guidelines, categorized into high, moderate, or low quality. High quality guidelines are those which has $\geq 60 \%$ score in three domains, including domain 3 (rigour of development). Moderate quality guidelines are categorized if the guideline has $\geq 60 \%$ score in three domains not including domain 3 . Those which didn't met the criterias are included in low quality guideline. The level of evidence (LOE) of recommendations from those guidelines was also compiled and stratified, formulated using standard LoE categorization by American College of Cardiology / American Heart Association (ACC/AHA) in 2016. ${ }^{9}$ Content analysis then made based on compilation of those recommendations. Matrix of relevant recommendation regarding asymptomatic carotid artery disease screening found in those 6 CPGs are then compiled and presented in Table 4, including LoE comparison between recommendations stated.

\section{RESULT}


Figure 1. Flowchart of the selected clinical practice guidelines

Keywords: asymptomatic, carotid artery disease OR carotid stenosis, ultrasound OR intimamedia thickness OR risk factor scoring, screening or guidelines

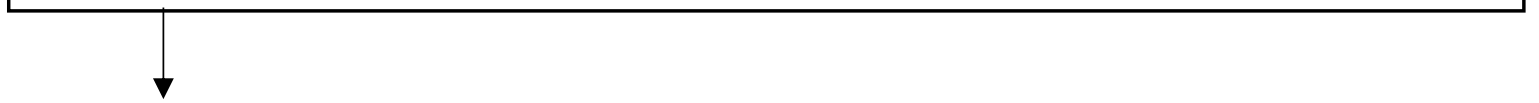

Studies identified using AHRQ, NICE, Cochrane Library, MEDLINE, EBSCO, and Proquest databases $(n=377)$

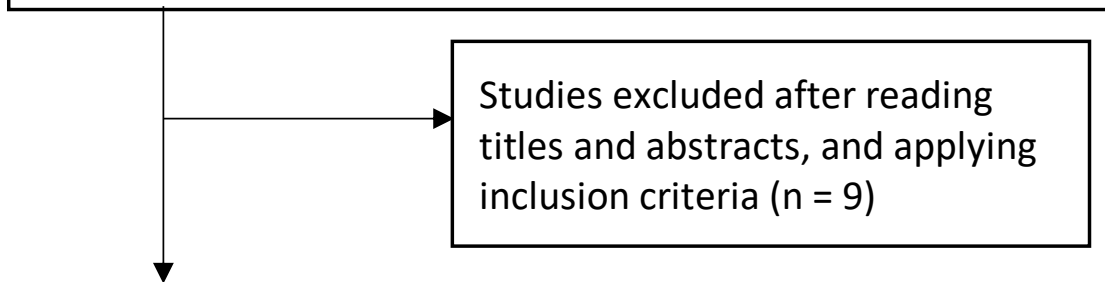

\section{Excluding same articles}

\section{3 articles excluded}

\section{Included guidelines $(\mathrm{n}=6)$}

The flowchart in Figure 1 shows the process by which we screened and selected the guidelines. Searching on and selecting CPGs from stated online databases were conducted and 6 CPGs were included in which the inclusion criteria were met. All the CPGs were written by profesional organizations based in the United States of America (USA) and Europe. Other characteristics of selected CPGs are presented in Table 2. Appraisal of CPGs using AGREE-II instrument was done and domain scores and quality stratification are presented in Table 3, showing that there is no CPGs classified as low-quality guidelines.

Most of those CPGs, precisely 5 of 6 CPGs, recommend screening evaluation by using ultrasound for asymptomatic high-risk population. Compiled from recommendations above, screening for carotid artery disease might be recommended for following population / patient, such as:

Symptomatic PAD.

Age more than 65 y.o. with 1 risk factor:

CAD, hypercholesterolemia, or tobacco smoking.

Having more than 2 risk factors: hypertension, hyperlipidemia, tobacco smoking, family history of ischaemic stroke, or early onset atherosclerosis.

CAD patients scheduled for CABG surgery with risk factor: aged $\geq 70$ y.o., abnormality in left main artery or multiple vessel disease, positive carotid bruit or PAD.

Permanent or transient retinal ischaemia without migraine or cardiac emboli.
Some recommendations in one CPG usually cited another CPG that also included in this study, which make recommendations from one CPG are usually similar to another. For example, recommendation given by Naylor et. al. is based on CPG written by Ricotta et. al and Brott et. al.

Those CPGs also clearly stated that routine screening for general population without atherosclerosis risk factor is not recommended. Carotid screening is only suggested for highly selective patients with several listed condition. It is only one CPG, from LeFevre et.al. which againsts carotid screening in asymptomatic population, with or without risk factor due to harm of screening outweigh the benefit. The study showed that by performing carotid artery screening, 217 number of stroke can be prevented in 5 years after 100,000 patients screened (Number needed to screen/NNS $=461$ ), which considered inefficient. Moreover, if estimated prevalence in general population screening was $5 \%$ positive and treated by surgical intervention accordingly, there will be 34 patients who will suffer cardiovascular or neurovascular event as surgical complication. 7,15

AGREE-II evaluation shows that 4 of 6 CPGs were categorized as high-quality guidelines, and two of them were moderate in quality (LeFevre et.al. and Qureshi et.al.). Two moderate-quality guidelines were classified based on inadequate score in domain 3 ( $\leq 60 \%$ score). Most of the recommendations, including in those which categorized into high-quality guidelines, are based on studies which ranged from LoE A to LoE C. 
Table 2. Characteristics of clinical practice guidelines included in the study

\begin{tabular}{|c|c|c|c|c|}
\hline Guidelines (Year) & Writer and organization & $\begin{array}{l}\text { Country or } \\
\text { region }\end{array}$ & $\begin{array}{l}\text { Level of } \\
\text { development }\end{array}$ & Status \\
\hline $\operatorname{ESC}(2017)^{11}$ & $\begin{array}{l}\text { Aboyans et.al.: European } \\
\text { Society of Cardiology }\end{array}$ & Europe & $\begin{array}{l}\text { Professional } \\
\text { organization }\end{array}$ & New \\
\hline USPSTF $(2014)^{7}$ & $\begin{array}{l}\text { LeFevre et.al.: United States } \\
\text { Preventive Services Task Force }\end{array}$ & USA & $\begin{array}{l}\text { Professional } \\
\text { organization }\end{array}$ & Updated \\
\hline AHA-ASA $(2011)^{12}$ & $\begin{array}{l}\text { Brott et.al.: American Heart } \\
\text { Association / American Stroke } \\
\text { Association }\end{array}$ & USA & $\begin{array}{l}\text { Professional } \\
\text { organization }\end{array}$ & New \\
\hline
\end{tabular}

Table 3. Domain scores (\%) of six clinical practice guidelines assesed using the AGREE-II instrument

\begin{tabular}{|c|c|c|c|c|c|c|}
\hline Domain & $\begin{array}{l}\text { ESVS } \\
2018\end{array}$ & $\begin{array}{l}\text { ESC } \\
2017\end{array}$ & $\begin{array}{l}\text { USPSTF } \\
2014\end{array}$ & $\begin{array}{l}\text { AHA-ASA } \\
2011\end{array}$ & $\begin{array}{l}\text { SVS } \\
2011\end{array}$ & ASN 2007 \\
\hline $\begin{array}{l}\text { Domain } \mathbf{1} \\
\text { Scope and purpose }\end{array}$ & 95.2 & 76.2 & 85.7 & 71.4 & 85.7 & 85.7 \\
\hline $\begin{array}{l}\text { Domain } 2 \\
\text { Stakeholder } \\
\text { involvement }\end{array}$ & 76.2 & 71.4 & 57.1 & 71.4 & 85.7 & 57.1 \\
\hline $\begin{array}{l}\text { Domain } 3 \\
\text { Rigor of development }\end{array}$ & 73.2 & 76.8 & 58.9 & 80.3 & 76.8 & 57.1 \\
\hline $\begin{array}{l}\text { Domain } 4 \\
\text { Clarity of } \\
\text { presentation }\end{array}$ & 100 & 71.4 & 71.4 & 80.9 & 95.2 & 100 \\
\hline $\begin{array}{l}\text { Domain } \mathbf{5} \\
\text { Applicability }\end{array}$ & 53.6 & 39.2 & 28.6 & 42.8 & 71.4 & 67.9 \\
\hline $\begin{array}{l}\text { Domain } 6 \\
\text { Editorial } \\
\text { independence }\end{array}$ & 71.4 & 92.3 & 57.1 & 92.8 & 85.7 & 35.7 \\
\hline Quality & High & High & Moderate & High & High & Moderate \\
\hline
\end{tabular}

\section{DISCUSSION}

As stated before, 5 of 6 guidelines recommend carotid artery screening only in high-risk asymptomatic population. Contradicting the others, LeFevre et.al. strongly against carotid screening in asymptomatic population, even when they already have several risks to develop ischaemic stroke. He stated that carotid screening, followed by next diagnostic evaluation and invasive treatments, deliver more harms to patients who are previously asymptomatic. Hypothetical outcomes stated in previous CPG of USPSTF shows that by carotid screening has relatively high NNS (217 ischaemic stroke prevented by screening 100.000 person). In the other hand, by performing surgical treatment for positively screened patient, 34 patients will possibly suffer from cardiovascular or neurovascular event as complication of the treatment, that might be avoidable if the screening wasn't performed in the first time. ${ }^{15}$ By those outcomes, the CPG stated that screening was not the effective way in preventing ischaemic stroke. More sophisticated risk assesment tools, not ultrasound screening, should be implemented in general or specific population. Unfortunately, the tools are not currently available yet. ${ }^{7,15}$

The results from carotid screening in specific population who are already in systemic atherosclerosis progression could give an alarm sign that the physician should increase the medication to prevent further progression more agressively using pharmacologic treatment, not necessarily proceed to surgical intervention by performing carotid artery stenting or endarterectomy. ${ }^{16}$ Hyperlipidemia, hypertension, tobacco smoking habit, and other risk factors that could worsen atherosclerosis progression further can be modified earlier as soon as the patients know they have asymptomatic carotid artery disease. Carotid stenosis and plaque that can be found by ultrasound examination could be a strong independent predictor of ischaemic stroke if not taken care seriously. ${ }^{16,17}$

Contradictive recommendations compared and evaluated by their LoEs. LeFevre et.al. againsts 
Table 4. Recommendation matrix of clinical practice guidelines included in the study

$\begin{array}{llll}\text { CPG source } & \text { CPG } & \text { Recommendations } & \text { Standardized } \\ & \text { quality } & & \begin{array}{l}\text { level of } \\ \text { evidence }\end{array}\end{array}$

Naylor et. al. High Selective screening for asymptomatic carotid stenosis may be $\mathrm{C}$

(ESVS 2018) quality considered in patients with multiple vascular risk factors to optimise risk factor control and medical therapy to reduce late cardiovascular morbidity and mortality, rather than for identifying candidates for invasive carotid interventions.

Aboyans et. High In patient with no recent ( $<6$ months) history of TIA/stroke, B

al. (ESC quality ultrasound screening may be considered in the following

2017) cases: age $\geq 70$ years, multivessel coronary artery disease

(CAD), concomitant lower extremity arterial disease (LEAD) or carotid bruit.

LeFevre et. Moderate The USPSTF recommends against all screening strategies,

al. (USPSTF quality including ultrasonography with or without confirmatory tests,

2014) for asymptomatic carotid artery stenosis in the general adult population (without history of TIA, stroke, or other neurologic signs or symptoms).

Brott et. al. High It is reasonable to perform duplex ultrasonography to detect

(AHA-ASA quality hemodynamically significant carotid stenosis in asymptomatic

2011) patients with carotid bruit.

Duplex ultrasonography to detect hemodynamically significant carotid stenosis may be considered in asymptomatic patients with symptomatic PAD, CAD, or atherosclerotic aortic aneurysm, but because such patients already have an indication for medical therapy to prevent ischemic symptoms. Duplex ultrasonography might be considered to detect carotid stenosis in asymptomatic patients without clinical evidence of atherosclerosis who have 2 or more of the following risk factors: hypertension, hyperlipidemia, tobacco smoking, a family history in a first-degree relative of atherosclerosis manifested before age 60 years, or a family history of ischemic stroke

\begin{tabular}{ll}
\hline Ricotta et. & High \\
al. (SVS & quality \\
$2011)$ &
\end{tabular}
Screening is not recommended for presence of a neck bruit A evidence 2011) Screening for asymptomatic clinically significant carotid bifurcation stenosis should be considered in certain groups of patients with multiple risk factors that increase the incidence of disease as long as the patients are fit for and willing to consider carotid intervention if a significant stenosis is discovered. Such groups of patients include: Patients with evidence of clinically significant peripheral vascular disease regardless of age; patients aged $\geq 65$ years with a history of one or more of the following atherosclerotic risk factors: CAD, smoking, or hypercholesterolemia.

Carotid screening may be considered in patients before coronary artery bypass graft (CABG) procedure. This is most likely to be fruitful if the patients are aged $\geq 65$ years and have left main disease or a history of peripheral vascular disease.

\begin{tabular}{ll}
\hline Qureshi et. & Moderate \\
al. (ASN & quality \\
2007) &
\end{tabular}

Screening of the general population or a selected population C

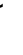
C based on age, gender, or any other variable alone is not recommended.

Screening of selected subpopulation aged 65 years or older with at least three cardiovascular risk factors (hypertension, $C A D$, current cigarette smoking, or hyperlipidemia) needs to be considered.

Screening of selected population undergoing coronary artery bypass grafting is strongly recommended. Selection criteria include age of 65 years or greater with either a history of stroke or TIA, left main coronary stenosis, PAD history of cigarette smoking, carotid bruit, previous carotid surgery, or diabetes mellitus.

Screening of all patients with symptomatic PAD is strongly recommended.

Screening for carotid artery stenosis is recommended for all patients with transient or permanent retinal ischemic event particularly in the absence of migraine or cardiac sources of emboli.

C

C
B
C
A
B


is categorized into LoE C, expert opinion, because recommendation was made based on hypothetical analysis, not by other qualified clinical studies. ${ }^{7,15}$ On the other hand, recommendation for performing screening in selective population were made by several high-quality studies, some of them are categorized into LoE A. Another reason is that although currently there is no available risk assesment tools mentioned by LeFevre et.al., all means of preventive treatment should be done in order to reduce the prevalence of ischaemic stroke. ${ }^{15}$ Ultrasound examination itself is widely available in Indonesia, relatively low cost, and non-invasive. ${ }^{18}$ By doing the examination, pharmacologic treatment, not necessarily surgery, can be adjusted to slow down the progression of atherosclerosis. ${ }^{16}$ Therefore, performing routine screening for specific population should be taken into consideration.

However, none of those CPGs recommend carotid artery IMT evaluation as an alternative of current worldwide standard, by measuring degree of stenosis of common carotid artery lumen. Although several studies deliver promising results showing that the IMT could shows earlier progression of systemic atherosclerosis and becoming one important predictor of cardiovascular and neurovascular complications, statistical analysis didn't yet strengthen the premise. ${ }^{16,17,18}$ IMT evaluation in carotid artery is expected to become a novel technique to evaluate earlier progression of ahterosclerotic disease systemically and locally in carotid artery, thus could earlier predict the risk of ischaemic stroke. It is now widely believed that increasing thickness of IMT by $0.1 \mathrm{~mm}$ may increase the risk of ischaemic stroke by
$13-18 \%$, and the discovery of this abnormal value may suggest physicians to increase the agressivity of medical treatment against systemic atherosclerosis progression. ${ }^{16}$ In spite of that, studies show that carotid stenosis and several standard ultrasound markers are now still statistically stronger predictors in diagnosing atherosclerosis carotid artery disease, and more studies evaluating IMT as sole predictor for ischaemic stroke's probability should be conducted to precisely measure the accuracy of proposed technique comparing current standard ultrasound evaluation. ${ }^{16,17}$

\section{CONCLUSION}

Performing carotid ultrasound screening for diagnosing carotid artery disease in high-risk populations might be recommended in order to prevent ischaemic stroke as neurovascular complication of systemic atherosclerosis. However current published CPGs don't recommend using carotid artery IMT as screening marker. Evaluating stenosis degree as standard ultrasound marker in diagnosis carotid artery disease is currently the only option.

\section{CONFLICT OF INTEREST}

The author states the original work, and there is no conflict of interest in doing this research.

\section{ORCID ID OF AUTHORS}

\section{REFERENCES}

1. Sobieszczyk P, Beckman J. Carotid artery disease. Circulation. 2006;114(7):244-248. doi:10.1161/CIRCULATIONAHA.105.542860.

2. Simova I. Intima-Media Thickness : Appropriate Evaluation and Proper. E-journal Cardiol Pract. 2015;13:1-14.

3. Azam M, Nida F, Handayani OWK, Fibriana AI. Lipid profiles and postprandial glucose status of hypertensive individuals with stroke in Indonesia. Asian J Epidemiol. 2017;10(3):116-122. doi:10.3923/aje.2017.116.122.

4. Shiber JR, Fontane E, Adewale A. Stroke registry : hemorrhagic vs ischemic strokes. Am J Emerg Med. 2010;28(3):331-333. doi:10.1016/j.ajem.2008.10.026.

5. Helmrich, Susan. Ragland, David. Leung, Rita. A.B. Paffenbarger R. Beneficial effect of carotid endarterectomy in symptomatic patients with high-grade carotid stenosis. N Engl J Med. 1994;325(3):147-152.

6. Darabian S, Hormuz M, Latif MA, Pahlevan S, Budoff MJ. The role of carotid intimal thickness testing and risk prediction in the development of coronary atherosclerosis. Curr Atheroscler Rep. 2013;15(3):1-6. doi:10.1007/s11883-012-0306-4.

7. LeFevre ML. Screening for asymptomatic carotid artery stenosis: U.S. Preventive Services Task Force recommendation statement. Ann Intern Med. 2014;161(5):356-362. doi:10.7326/M14-1333.

8. Brouwers MC, Kerkvliet K, Spithof K. The AGREE reporting checklist: A tool to improve reporting of clinical practice guidelines. BMJ. 2016;352. doi:10.1136/bmj.i1152.

9. Halperin JL, Levine GN, Al-Khatib SM, et al. Further evolution of the ACC/AHA clinical practice guideline recommendation classification system: A report of the American College of Cardiology/American Heart Association Task Force on Clinical Practice Guidelines. Circulation. 2016;133(14):1426-1428. doi:10.1161/CIR.0000000000000312.

10. Naylor AR, Ricco JB, Borst GJ, et al. Re: "Editor's Choice - Management of Atherosclerotic Carotid and Vertebral Artery Disease: 2017 Clinical Practice Guidelines of the European Society for Vascular Surgery (ESVS)." Eur J Vasc Endovasc Surg. 2018;55(6):901-902.

11. Aboyans V, Ricco JB, Bartelink MLEL, et al. 2017 ESC Guidelines on the Diagnosis and Treatment of Peripheral Arterial Diseases, in collaboration with the European Society for Vascular Surgery (ESVS). Eur Heart J. 2018;39(9):763-816. doi:10.1093/eurheartj/ehx095.

12. Brott Halperin JL, Abbara S, et $\begin{array}{r}\text { al. } \\ \text { ASA/ACCF/AHA/AANN/AANS/ACR/ASNR/CNS/SAIP/SCAISIR/SNIS/SVM/SVS guideline on the }\end{array}$ management of patients with extracranial carotid and vertebral artery disease: Executive summary. J Am Coll Cardiol. 2011;57(8):1002-1044. doi:10.1016/j.jacc.2010.11.005.

13. Ricotta JJ, Aburahma A, Ascher E, Eskandari M, Faries P, Lal BK. Updated Society for Vascular Surgery 
guidelines for management of extracranial carotid disease. J Vasc Surg. 2011;54(3):e1-e31. doi: $10.1016 / j . j v s .2011 .07 .031$.

14. Qureshi AI, Alexandrov A V., Tegeler CH, Hobson RW, Dennis Baker J, Hopkins LN. Guidelines for screening of extracranial carotid artery disease: A statement for healthcare professionals from the Multidisciplinary Practice Guidelines Committee of the American Society of Neuroimaging; cosponsored by the society of vascular and interven. J Neuroimaging. 2007;17(1):19-47. doi:10.1111/j.15526569.2006.00085.x.

15. Wolff T, Shelton E, Sessions C, Miller T. Screening for Carotid Artery Stenosis: An Update of the Evidence for the US Preventive Service Task Force. Ann Intern Med. 2007;150(10):710-716.

16. Van den Oord SCH, Sijbrands EJG, ten Kate GL, et al. Carotid intima-media thickness for cardiovascular risk assessment: Systematic review and meta-analysis. Atherosclerosis. 2013;228(1):1-11. doi:10.1016/j.atherosclerosis.2013.01.025.

17. Yoon $\mathrm{HJ}$, Kim $\mathrm{KH}$, Park $\mathrm{H}$, et al. Carotid plaque rather than intima-media thickness as a predictor of recurrent vascular events in patients with acute ischemic stroke. Cardiovasc Ultrasound. 2017;15(1):1-8. doi:10.1186/s12947-017-0110-y.

18. Wyman RA, Fraizer MC, Keevil JG, et al. Ultrasound-detected carotid plaque as a screening tool for advanced subclinical atherosclerosis. Am Heart J. 2005;150(5):1081-1085. doi:10.1016/j.ahj.2005.01.010

19. Darabian S, Hormuz M, Latif MA, Pahlevan S, Budoff MJ. The role of carotid intimal thickness testing and risk prediction in the development of coronary atherosclerosis. Curr Atheroscler Rep. 2013;15(3):16. doi: 10.1007/s11883-012-0306-4 